\title{
ON THE CENTRAL LIMIT THEOREM IN $F$-SPACES
}

\author{
WERNER E. HELM
}

\begin{abstract}
In this note we improve the known result on the integrability of $F$-norms with respect to Gaussian measures and obtain a Central Limit Theorem for probability measures on arbitrary separable $F$-spaces.
\end{abstract}

1. Let $E$ be a real linear space. A function $\|\cdot\|: E \rightarrow[0, \infty)$ is called $F$-seminorm if it satisfies

(i) $\|x+y\| \leqslant\|x\|+\|y\|, x, y \in E$,

(ii) $\|\alpha x\| \leqslant\|x\|, \alpha \in \mathbf{R},|\alpha| \leqslant 1, x \in E$,

(iii) $\|\alpha x\| \rightarrow 0, \alpha \in \mathbf{R}, \alpha \rightarrow 0, x \in E$.

$\|\cdot\|$ is an $F$-norm if $\|x\|=0$ implies $x=0$ and it is called $p$-homogeneous, $0<p \leqslant 1$, if $\|\alpha x\|=|\alpha|^{p}\|x\|$ for $\alpha \in \mathbf{R}, x \in E$. Every $F$-norm defines a translation invariant metric $d(x, y)=\|x-y\|$ which makes $E$ into a linear metric space. If $E$ is complete with respect to $d(x, y)$ we call it an $F$-space. We will consider separable $F$-spaces only. Provided with its Borel $\sigma$-algebra $\mathfrak{B},(E, \mathfrak{B})$ then becomes a measurable linear space in the sense of Fernique [2], whose definition of a Gaussian measure we adopt. Let the mappings $T_{s, t}$ : $E \times E \rightarrow E \times E$ be defined as

$$
T_{s, t}(x, y)=(s x-t y, t x+s y), \quad s, t \in \mathbf{R} .
$$

We say that a probability measure $\subseteq$ on $E$ is mean zero Gaussian if the product measure $\subseteq \otimes \subseteq$ is invariant with respect to the family $\left\{T_{s, t}: s^{2}+t^{2}\right.$ $=1\}$.

Concerning the integrability of $F$-norms with respect to Gaussian measures we have the following fundamental result.

THEOREM 1. Let $(E,\|\cdot\|)$ be a separable $F$-space with p-homogeneous $F$-norm, $0<p \leqslant 1$, let $r=2 /(2-p)$ and $\subseteq$ be a mean zero Gaussian measure on $(E, \mathfrak{B})$. Then there exists an $\varepsilon>0$ such that

$$
\int \exp \left(\varepsilon\|x\|^{r}\right) \subseteq(d x)<\infty
$$

We omit the proof since it follows from a careful inspection and adaptation of Fernique's original proof [2] as indicated in [4] for $r=1$. We only remark that the theorem still holds in the more general situation, when $(E, \mathfrak{B})$ is just a measurable linear space and $\|\cdot\|$ a measurable pseudo- $F$-seminorm, which satisfies $S(x:\|x\|<\infty)>0$.

Received by the editors September 10, 1977 and, in revised form, March 27, 1978.

AMS (MOS) subject classifications (1970). Primary 60B10, 60F05, 60G15.

Key words and phrases. Gaussian measures on $F$-spaces, integrability of $F$-norms, Central Limit Theorem.

- American Mathematical Society 1978 
2. If $E^{*}$, the topological dual of $E$, separates points of $E$, our definition of Gaussian measures coincides with the usual one: $\mathbb{S}$ is mean zero Gaussian if and only if every $f \in E^{*}$ is a (possibly degenerate) normally distributed real random variable with zero mean on $(E, \mathfrak{B}, \mathcal{S})$. In this case we assume throughout all probability measures $\mu$ to be weakly centered, i.e.

$$
\int f(x) \mu(d x)=0, \quad f \in E^{*} .
$$

If $\mu$ has weak second moment, i.e. $E^{*} \subseteq L^{2}(E, \mathfrak{B}, \mu)$, then

$$
\Gamma_{\mu}(f, g)=\int f(x) g(x) \mu(d x), \quad f, g \in E^{*}
$$

will denote the covariance of $\mu$, and we call $\mu$ pregaussian if it has the same covariance as some Gaussian measure $\subseteq$. By $\mathcal{L}(X)$ we denote the distribution of a random element $X$ defined on some probability space with values in $E$. Finally we say that $\mu$ satisfies the Central Limit Theorem (CLT) if the distributions of $\sqrt{n}^{-1}\left(X^{(1)}+\cdots+X^{(n)}\right)$ converge $\|\cdot\|$-weakly to a Gaussian measure $\subseteq$ on $E$, where $X^{(j)}$ are independent copies of $X, \mathcal{L}(X)=$ $\mu$.

In [3] we had investigated Gaussian measures and the CLT in certain Orlicz sequence spaces determined by a sequence of subadditive Orlicz functions as follows

$$
l_{\phi}=\left\{\left(y_{j}\right): y_{j} \in \mathbf{R}, \sum_{j} \varphi_{j}\left(\left|y_{j}\right|\right)<\infty\right\}, \quad\|y\|_{\phi}=\sum_{j} \varphi_{j}\left(\left|y_{j}\right|\right) .
$$

In the study of measures on these sequence spaces a basic role is played by the so called "standard deviation vector" $\sigma(\mu)$ defined coordinatewise as

$$
(\sigma(\mu))_{j}=\Gamma_{\mu}\left(e_{j}^{*}, e_{j}^{*}\right)^{1 / 2}=\left(\int y_{j}^{2} \mu(d y)\right)^{1 / 2}, \quad j=1,2, \ldots,
$$

$\left\{e_{j}, e_{j}^{*}\right\}$ denoting the canonical basis of $l_{\phi}$.

For our present purpose the results of [3] may be summarized as follows.

THEOREM 2. (i) A probability measure $\mu$ on $l_{\phi}$ is pregaussian if and only if $\sigma(\mu) \in l_{\phi}$.

(ii) $A$ probability measure $\mu$ on $l_{\phi}$ satisfies the CLT if and only if it is pregaussian.

Further results emphasizing the importance of the standard deviation vector will be published elsewhere.

Now we give a sufficient condition for the CLT to hold in an arbitrary separable $F$-space $(E,\|\cdot\|)$.

TheOREM 3. Let $X=\sum_{i} \xi_{i}(\omega) x_{i}, \sigma \neq x_{i} \in E$, $\xi_{i}$ real random variables, be a.s. absolutely convergent, $\mu=\mathcal{L}(X)$. If the variables $\xi_{i}$ have zero expectations, finite second moment and 


$$
\sum_{i}\left\|\sigma\left(\xi_{i}\right) x_{i}\right\|<\infty
$$

then $\mu$ satisfies the Central Limit Theorem in $E$.

Proof. Setting $\varphi_{j}(t)=\left\|t x_{j}\right\|, t>0, j=1,2, \ldots$, we obtain subadditive Orlicz functions in the above sense. Denoting by $l_{\phi}$ the corresponding Orlicz sequence space

$$
v: l_{\phi} \rightarrow E, \quad v\left(\left(\eta_{j}\right)\right)=\sum_{j} \eta_{j} x_{j}
$$

becomes a linear and continuous map. Now $\xi(\omega)=\left(\xi_{1}(\omega), \xi_{2}(\omega), \ldots\right)$ defines a random element with values in $l_{\phi}$ which is pregaussian and satisfies the CLT in $l_{\phi}$ because of Theorem 2 and condition (*). Hence there exists a Gaussian measure $\subseteq$ on $l_{\phi}$ such that $\theta_{n}\left(\nu^{n^{*}}\right)$ converges $\|\cdot\|_{\phi}$-weakly to $\subseteq$, where $\theta_{n} x=(1 / \sqrt{n}) x, \nu$ is the distribution of $\xi, \nu^{n^{*}}$ its $n$-fold convolution. Since $v$ is continuous, it follows that $v(\Im)$ is Gaussian on $E$ and that $\theta_{n}\left(\mu^{n^{*}}\right)$ converges $\|\cdot\|$-weakly to $v(\Im)$.

One should remark that we did not assume the variables $\xi_{i}$ to be independent. Moreover, choosing the $x_{i}$ to form a countable dense subset of $E$, the corresponding map $v$ becomes onto [5] and it follows that every probability measure $\mu$ on $E$ can be obtained as $\mu=\mathcal{L}(X), X$ absolutely convergent as presumed. However, due to its general nature, condition (*) is quite strong in many spaces. But according to Theorem 2, if $x_{j}=e_{j}$, it is necessary in all sequence spaces $l_{\phi}$, including the Banach space $l^{1}$. Hence condition (*) cannot be weakened without restricting the class of spaces for which Theorem 3 is valid. As far as only Banach spaces are concerned, Theorem 3 could of course be obtained using [1, Theorem 6.1] instead of Theorem 2 .

ACKNOWLEDGEMENT. I would like to thank the referee for his useful comments on the presentation and final form for this note.

\section{REFERENCES}

1. A. D. de Acosta, Existence and convergence of probability measures in Banach spaces, Trans. Amer. Math. Soc. 152 (1970), 273-298.

2. X. Fernique, Intégrabilité des vecteurs gaussiens, C. R. Acad. Sci. Paris 270 (1970), 1698-1699.

3. W. E. Helm, Gaussian random elements in certain Orlicz sequence spaces, Bull. Acad. Polon. Sci. 25 (1977), 507-514.

4. T. Inglot and A. Weron, On Gaussian random elements in some non-Banach spaces, Bull. Acad. Polon. Sci. 22 (1974), 1039-1043.

5. Ph. Turpin, Convexités dans les espaces vectoriels topologiques généraux, Thèse, Orsay, 1974.

Fachibreich Mathematix, Technische Universität Berlin, D-1000 Berlin 12, Federal REPUBLIC OF GERMANY 